Estudios Pedagógicos XXXIX, Número Especial 1: 121-141, 2013

INVESTIGACIONES

\title{
La experiencia Kelluwen: Tres años de desarrollo y puesta en práctica de una propuesta de innovación didáctica con uso de TIC
}

\author{
The Kelluwen Experience: Three years in the development and practice of an approach of \\ didactic innovation using ICT
}
Kelluwen Experiência: Três anos de desenvolvimento e implementação de uma inovação educacional com o uso das TIC

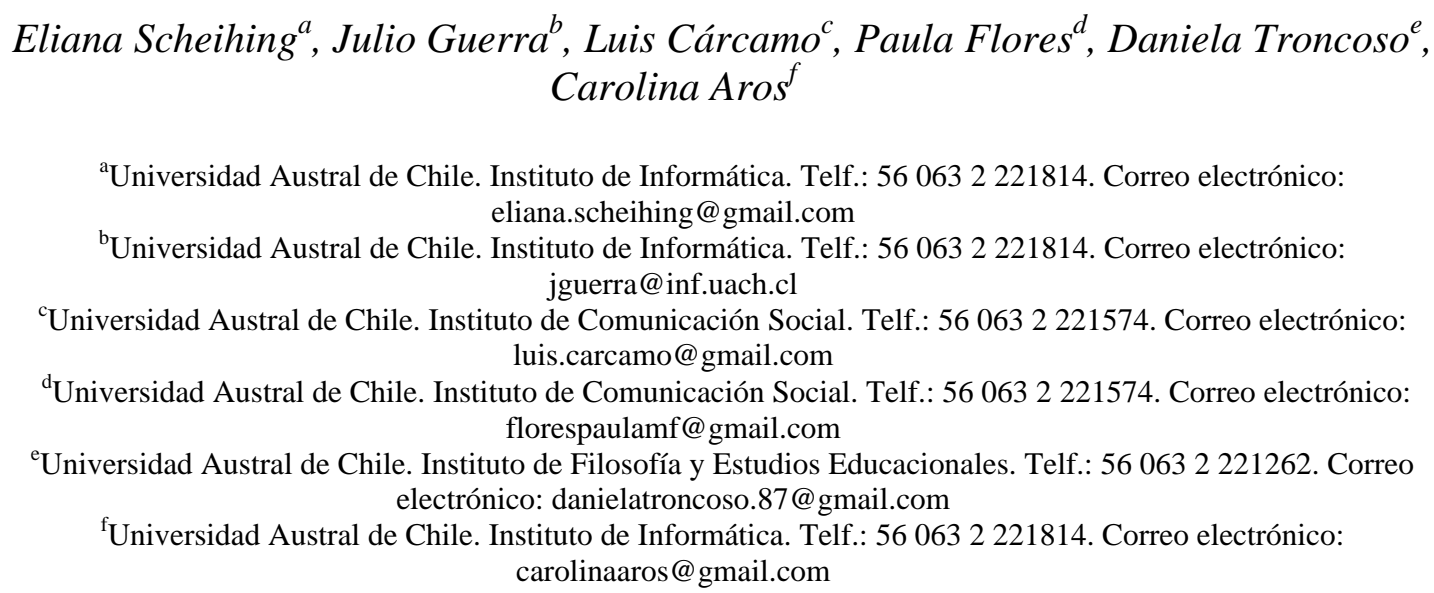

RESUMEN

Kelluwen ha validado una estrategia didáctica integral de inserción curricular de las TIC en el aula, orientada al mejoramiento de competencias socio-comunicativas en estudiantes de 12 a 16 años. La estrategia didáctica se sustenta en el constructivismo social y se despliega en tres ejes: creación y validación de 17 Diseños Didácticos Colaborativos que utilizan la Web Social, desarrollo de plataforma Web Kelluwen y desarrollo de una Estrategia Evaluativa Integral. La experiencia en 167 aulas de escuelas y liceos vulnerables del sur de Chile arroja resultados promisorios. En el ámbito cuantitativo, el análisis de una muestra de 3 aulas indica una mejora significativa en las habilidades lecto-escriturales, con un $63 \%$ de estudiantes que aumentan su índice Cloze, frente al $45 \%$ en el aula control. En el ámbito cualitativo, los estudiantes evalúan positivamente la experiencia Kelluwen, asociándola al aumento en sus niveles de apropiación, protagonismo y motivación en el proceso de aprendizaje.

Palabras Clave: innovación educativa, didáctica 2.0, web social, web 2.0, sur de Chile

\begin{abstract}
Kelluwen validated a holistic didactical strategy incorporating the use of ICT in classroom curricula aiming to improve socio-communicative skills in students from 12 to 16 years old. The didactical strategy is based on the socio-constructivist approach and it is deployed in three axes: the validation of 17 Collaborative Didactical Designs, the development of the Kelluwen Web Platform, and the development of an integral evaluation strategy. The experience in 167 classrooms in schools of southern Chile showed promising results. In the quantitative scope, the analysis of the Cloze test from a sample of 3 classrooms showed a significant
\end{abstract}


improvement of the literacy skills in $63 \%$ of the students compared to $45 \%$ in the control classroom. In the qualitative scope, the students assessed the learning activities as positive, and the results showed an association with the increase in their levels of ownership, leadership and motivation in the learning process.

Keywords: educational innovation, didactic 2.0, social web, web 2.0, southern Chile

\section{RESUMO}

Kelluwen têm validado uma estratégia didática integral de inserção curricular das TIC na sala de aula, orientada ao melhoramento das competências sócio-comunicativas em estudantes de 12 até 16 anos. A estratégia didática sustenta-se no construtivismo social e estende-se em três eixos: criação e validação de 17 Modelos Didáticos Colaborativos que utilizam a Web Social, desenvolvimento da plataforma Web Kelluwen e o desenvolvimento de uma estratégia avaliativa integral. A prática em 167 salas de aula de escolas e liceus vulneráveis no sul do Chile permite observar resultados prometedores. No âmbito quantitativo, a análise de uma mostra de 3 salas de aula indica uma melhoria significativa nas habilidades da lectoescritura, num $63 \%$ de estudantes que aumentam o seu índice Cloze, frente aos $45 \%$ na sala de aula de controle. No âmbito qualitativo, os estudantes avaliam positivamente a prática Kelluwen, numa associação ao aumento nos seus níveis de apropriação, protagonismo e motivação no processo da aprendizagem.

Palavras chave: inovação educativa, didática 2.0, web social, web 2.0, sul do Chile.

\section{INTRODUCCIÓN}

\subsection{LOS DESAFÍOS EDUCATIVOS EN LA SOCIEDAD RED}

La escuela, en la actual sociedad de la información y del conocimiento, se enfrenta al desafío de cambiar el antiguo paradigma pedagógico en el cual los profesores se limitaban a ser meros transmisores de conocimientos (Hargreaves y Fullan, 2012). En los nuevos tiempos, la educación formal como institución ha perdido la exclusividad del proceso de enseñanza aprendizaje. El computador e Internet en los hogares permiten que las personas tengan la información en sus casas, por tanto, entregar información deja de ser un rol hegemónico de las escuelas y los medios de comunicación de masas; pero sí importa qué hacer con ésta o cómo utilizarla (Arancibia, 2004). El desafío se hace mayor al considerar los cambios paradigmáticos asociados con la Web Social o Web 2.0. Contreras et al. (2009) evidencian que este cambio transforma sustancialmente la forma de interactuar, en donde se transita desde una Web dedicada exclusivamente a la lectura hacia una que mezcla la lectura y la escritura, la creación y la publicación. De lectores se pasa a ser autores. En educación, las transformaciones sociales paradigmáticas implican un nuevo modo de conocer, y por tanto de aprender. Así, el constructivismo social gana adeptos sobre la enseñanza transmisiva (Aubert et al., 2011) e impone nuevas ideas. Es así como en la actualidad, al momento de realizar una propuesta educativa de uso de TIC, hay que considerar la Web 2.0 (Area y Ribeiro, 2012).

Por otra parte, la integración pedagógica de las TIC en centros escolares, especialmente en las aulas escolares, a menudo se ha constituido en un proceso complicado, aún no logrado (Arancibia et al., 2010; Sigalés et al., 2009). Según Enlaces, "diversos estudios mostraron un muy bajo grado de transformación de las prácticas en las escuelas y las aulas, y los propios actores del sistema percibían el pobre impacto de las políticas implementadas" (Bilbao y Salinas, 2010: 81). 
Estudios Pedagógicos XXXIX, Número Especial 1: 111-131, 2013 LA EXPERIENCIA KELLUWEN: TRES AÑOS DE DESARROLLO Y PUESTA EN PRÁCTICA DE UNA

\subsection{EL USO DE TIC EN EL SISTEMA EDUCATIVO CHILENO}

A partir del año 1996, se diseña en Chile una política de Estado para implementar el uso de las TIC en el aula. Específicamente, este proceso comenzó con el Proyecto Enlaces que intervino en el desarrollo profesional docente, así como también en la cooperación para la modernización del sistema escolar. El Proyecto Enlaces fue un aporte a la reducción de la brecha digital de las nuevas generaciones (Hepp, 2003). A mediados de la década pasada, se hizo presente la necesidad de una renovación curricular en la formación inicial y continua de profesores, detectando la falta de manejo de los docentes en el uso de las TIC para la enseñanza. De esta manera, el Ministerio de Educación comienza a discutir sobre las competencias y estándares TIC para la formación de profesores (Mineduc, 2009) y en el 2010 se propone el Marco de Competencias Tecnológicas para el Sistema Escolar. El proceso de implementación de las TIC en las escuelas de Chile se constituye en una experiencia exitosa y reconocida internacionalmente, logrando implementar tecnología en las escuelas y a su vez capacitando masivamente a los docentes en el uso instrumental básico de estos recursos. Estudios recientes sobre nuevas iniciativas del programa Enlaces, como es el caso de los Laboratorios Móviles Computacionales (Nussbaum et al. 2012), muestran que para la inclusión didáctica de las TIC en aula existen dos grandes obstáculos: el escaso acompañamiento pedagógico y técnico sistemático al profesor de aula, y la falta un compromiso integral de los centros escolares en el proceso de innovación educativa. En este mismo sentido, un estudio desarrollado en Chile (Salinas et al., 2009) para detectar los factores que influyen en el desarrollo y sustentabilidad de prácticas innovadoras de integración curricular de las TIC en aulas de Enseñanza Media, señala que un factor relevante en dicha sustentabilidad es que los docentes cuenten con el apoyo y reconocimiento de su centro escolar. Posteriormente, este mismo autor desarrolla un estudio comparativo de la realidad chilena respecto de la participación de los profesores en redes profesionales digitales e innovación en las prácticas docentes en la sala de clases (Salinas et al. 2012), concluyendo que la mayoría de las redes existentes en Chile tiene un carácter incipiente, y que los profesores las usan mayoritariamente como un espacio para la búsqueda de información y/o material didáctico y, en menor grado, como un espacio de intercambio de experiencias.

\subsection{COMPETENCIAS SOCIO-COMUNICATIVAS Y DIGITALES}

El acto de enseñanza aprendizaje es también un acto de comunicación (Kaplun, 1998) en el que intervienen actores dentro y fuera del aula. Cuando un niño o un adolescente ejecuta una tarea en la sala de clases está interactuando directamente con su profesor y algunos de sus compañeros; y además el producto de esa tarea o evidencia de aprendizaje específica será un mensaje que -más allá del esfuerzo intelectual- busca ser comunicado a su comunidad de estudio. Si todo acto de aprendizaje es un acto de comunicación, no es difícil suponer que el lenguaje es el articulador de cualquier escena didáctica. La baja comprensión lectora, la deficiente producción textual y el escaso pensamiento crítico son parte de una raíz común de problemas de aprendizajes escolares. En la actualidad, parece indispensable desarrollar competencias socio-comunicativas entendidas como habilidades que permitan a los individuos asumir compromisos de interacción, considerar normas elementales contextuales para establecer relaciones con 
otros y la comprensión de los diferentes signos, significados y códigos presentes en los contextos socioculturales. En una mirada actualizada, al igual que Coll (2005), consideramos que el hipertexto y la Web vienen a potenciar nuevas habilidades en lo referente a comprensión lectora y producción de texto, y por tanto son nuevas oportunidades de aprendizaje.

\subsection{UN CAMBIO POSIBLE: LA DIDÁCTICA 2.0}

Después de tres años de experiencia en el desarrollo de innovación educativa usando TIC en más de 160 aulas en el sur del país, el equipo de investigación Kelluwen ha acuñado el concepto de "Didáctica 2.0". Este concepto se refiere a la acción pedagógica que tiene sus principios en el constructivismo social y que se basa en el modo de hacer de los jóvenes en la Web 2.0, esto es: crear y publicar contenido, compartirlo, valorarlo y generar reflexión a partir de la retroalimentación recibida. La idea es incorporar estos elementos en el tratamiento de las unidades de contenido abriendo espacios para el diálogo, la crítica, la retroalimentación y la reflexión a partir del producto o evidencia de aprendizaje que es creado y publicado en la Web por los mismos estudiantes. El aprendizaje visto así es un ejercicio asociado a la capacidad de los estudiantes de elaborar un producto o evidencia de aprendizaje de carácter público, que adquiere visibilidad en la Web 2.0. Por ejemplo, un video realizado por estudiantes de la ciudad de Valdivia puede ser visualizado y comentado por estudiantes de la localidad de Hualaihué.

La propuesta Kelluwen implementa la idea de Didáctica 2.0 con tres componentes principales: a) el Diseño Didáctico Colaborativo (DDC), que incorpora la unidad de contenido y el material, organiza las actividades de aprendizaje y sugiere los momentos para producir, compartir, dialogar y reflexionar; b) la plataforma Web Kelluwen, que apoya la ejecución de los DDC e incorpora herramientas para facilitar la comunicación y colaboración entre profesores y estudiantes entre e intra aulas; c) una metodología en espiral de desarrollo en donde los DDC y la plataforma Web son iterativamente mejorados a partir de la observación de su uso en aulas y del testimonio de los profesores y alumnos. Esta metodología ha permitido mejorar las propuestas de intervención (en consideración tanto de las actividades didácticas, como de la plataforma Web) rescatando aspectos clave para su buen desempeño en el contexto real de aula desde los mismos actores (profesores y estudiantes).

En la sección siguiente, se exponen los principales elementos de un DDC y su implementación a través del uso de la plataforma Kelluwen. Luego se presentan resultados de tres años de experiencia Kelluwen, y se finaliza con la discusión y conclusiones de estos resultados.

\section{LOS DISEÑOS DIDÁCTICOS COLABORATIVOS Y SU DESPLIEGUE EN LA PLATAFORMA KELLUWEN}

\subsection{ELEMENTOS METODOLÓGICOS DE LA PROPUESTA}

La propuesta didáctico-pedagógica de Kelluwen, denominada Diseños Didácticos Colaborativos (DCC) (Scheihing et al., 2011), busca articular el trabajo de aula para profesores interesados en innovar con herramientas de la Web Social. Se trata de abordar 
contenidos curriculares regulares incorporando un uso creativo y dialógico de los recursos de Internet. Es por ello que cada diseño se apropia de una aplicación de la Web Social (Youtube, Wordpress, Facebook, Panoramio, Gigapan, entre otros) para convertir estas herramientas, tan cercanas de los estudiantes, en parte activa del escenario pedagógico en sus aulas. La principal motivación para el uso de este tipo de herramientas en aula es la característica inherentemente social de las mismas, en donde el trabajo de los usuarios, en este caso la evidencia de aprendizaje los estudiantes, queda disponible en forma pública y puede ser accedido, comentado e incluso modificado por otros.

Estructura. Cada Diseño Didáctico Colaborativo (DDC) está centrado en una unidad de contenido curricular y considera un periodo de ejecución de 8 a 12 sesiones combinando el uso del aula regular, laboratorio y salidas a terreno. Las sesiones se organizan en tres etapas: Motivación, Creación y Evaluación. Estas etapas son descritas más adelante en la sección 2.3. Cada sesión se compone de 3 momentos: inicio, desarrollo y cierre. Estos momentos permiten maximizar el uso del tiempo y definir las estrategias y recursos necesarios para lograr actividades de aprendizaje satisfactorias que involucren y motiven a todos los estudiantes (Mineduc, 2003). El inicio de la clase se constituye en el momento de integrar a los estudiantes al proceso educativo, recuperar los conocimientos previos, encadenar la actividad actual con la actividad previa del DDC y concretar un estado de motivación que comprometa a los estudiantes a ser protagonistas de su aprendizaje. En el momento de desarrollo, se orienta a los estudiantes a que descubran y construyan conocimiento apropiándose del objeto de estudio que conocerán, proceso que es sostenido a partir de los aprendizajes esperados propuestos para cada clase. El momento de cierre de la clase está orientado a promover una instancia de reflexión, evaluación y síntesis respecto a las actividades desarrolladas. En este momento se procura que los estudiantes formen una visión acerca de qué aprendieron y cuál es la utilidad de las estrategias y experiencias desarrolladas para promover su aprendizaje, así como también visualizar la actividad próxima.

Colaboración. La colaboración, columna vertebral de la propuesta, se propicia en dos espacios. Los alumnos trabajan en grupos de entre 3 y 5 estudiantes, y además interactúan con aulas gemelas, que son otras aulas escolares que ejecutan el mismo DDC en otro punto geográfico del país. El DDC direcciona una dinámica de colaboración y revisión de pares promoviendo que las aulas gemelas valoren y opinen sobre las evidencias de aprendizaje de otros estudiantes.

Contextualización. Los DDC son capaces de integrar las particularidades que presentan los estudiantes y su entorno socio-cultural potenciándose, de este modo, como actividades relevantes para ellos (Álvarez, 2009). Es así como matices provenientes desde variabilidades etarias, procedencia urbana o rural, nivel de enseñanza, o intereses de ocio y cultura tales como grupos musicales, películas, comunidades virtuales de interés, pueden aportar a la creación de evidencias de aprendizaje más pertinentes y significativas para los y las estudiantes.

Compromiso. El compromiso de los estudiantes en el proceso de enseñanza aprendizaje es fundamental para garantizar un desarrollo efectivo de las evidencias de aprendizaje, y también para generar una evaluación auténtica centrada en los procesos individuales y colectivos ejecutados a lo largo de la experiencia didáctica (Álvarez, 2009). En los DDC, cada integrante de grupo tiene un rol. Por ejemplo, en un grupo pueden articularse funciones de administrador informático, editor de contenidos e 
investigador/historiador. Esta responsabilidad permite la apropiación del proceso de aprendizaje debido a que cada estudiante es una parte fundamental de su equipo de trabajo, tanto en la construcción de la evidencia de aprendizaje como en la evaluación constante de las actividades que se desarrollan.

Rol del docente. Si bien, en la planificación de un DDC se puede observar un rol protagónico de los estudiantes, puesto que son éstos quienes se enfrentan al proceso de descubrimiento y construcción del conocimiento, debemos destacar el rol mediador que ejerce cada profesor/a. El profesor tiene un rol central, distinto a la transmisión de conocimientos, que involucra varias funciones: motiva y orienta las actividades que son propuestas; adapta la temática a la realidad contextual, cultural y geográfica de la clase; media y coordina la interacción de los estudiantes cuando es necesario; y asiste las necesidades particulares de los estudiantes.

\subsection{EL ROL DE LA PLATAFORMA KELLUWEN}

La propuesta metodológica de los Diseños Didácticos Colaborativos se apoya en la plataforma Web Kelluwen. Esta herramienta incorpora una serie de módulos de gestión, comunicación y coordinación para apoyar las interacciones de profesores y estudiantes en el desarrollo de las actividades didácticas y en el uso de las herramientas de la Web Social. Un módulo de micro-blogging denominado Bitácora (ver Figura 1) permite a los estudiantes interactuar durante la clase de laboratorio con sus compañeros y con el profesor, y al mismo tiempo contar con un registro del desarrollo de la clase que puede ser utilizado por el profesor en una posterior evaluación (Inalef et al., 2012).

Figura 1. Ejemplo de uso del módulo Bitácora de la plataforma Kelluwen

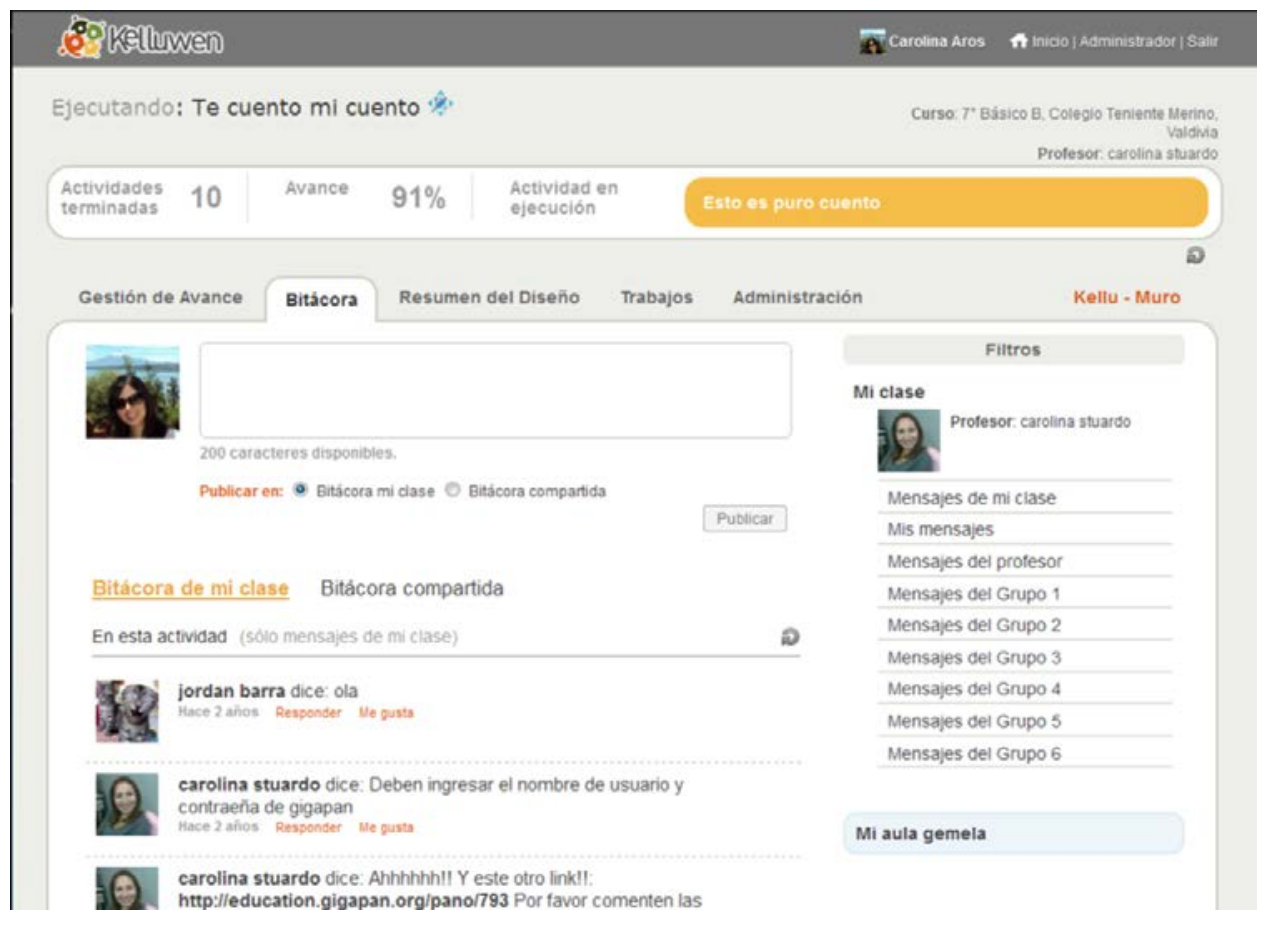


En el módulo de Gestión de Avance de la plataforma, el profesor coordina el avance de las actividades didácticas y accede al material respectivo. Los estudiantes también tienen la posibilidad de ver la planificación completa del DDC, y de revisar el registro de interacciones -conversaciones- pasadas. En la Figura 2 se presenta una vista del módulo de Gestión de Avance con cada una de las etapas de la planificación didáctica. En esa figura se encuentra seleccionada la etapa de Creación para la cual está visible cada una de las sesiones que la componen con su descripción y lista de materiales disponibles. En la Figura 3 se presenta la descripción de una sesión en particular, que incluye los aprendizajes esperados y las evidencias de la sesión, así como una descripción de los tres momentos de la clase.

En el módulo de Trabajos (ver Figura 4) los estudiantes publican las evidencias de aprendizaje desarrolladas en una herramienta de la Web Social (por ejemplo, Youtube). De esta forma, los trabajos son asociados a los grupos de un aula y son accesibles a otras aulas. El módulo incorpora un mecanismo de asignación automática para la co-evaluación por parte de dos grupos de estudiantes de aulas gemelas o, en su defecto, de la misma aula.

Figura 2. Módulo de Gestión de Avance con las Etapas del Diseño Didáctico

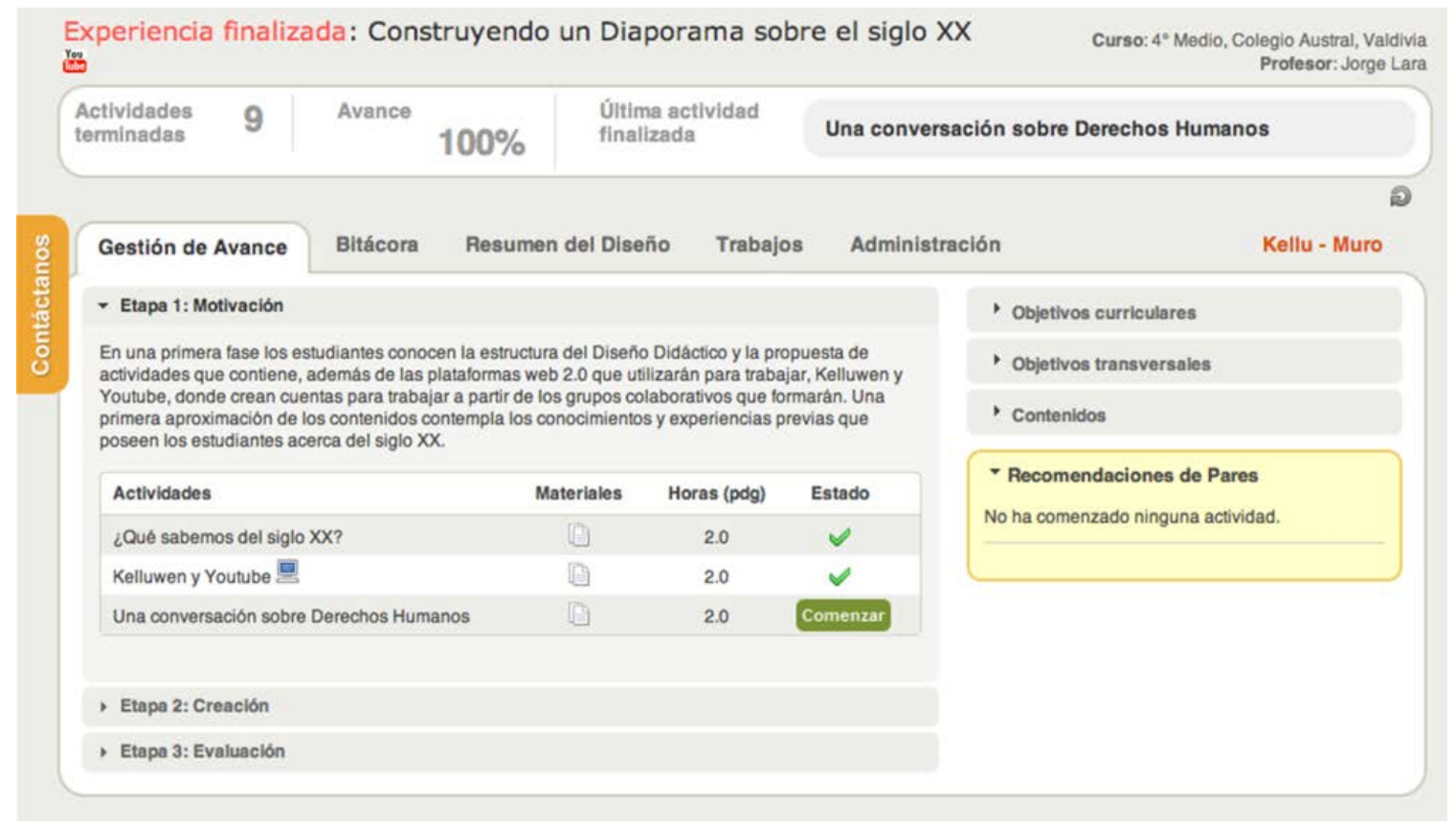


Figura 3. Descripción de una sesión en módulo de Gestión de Avance de la plataforma.

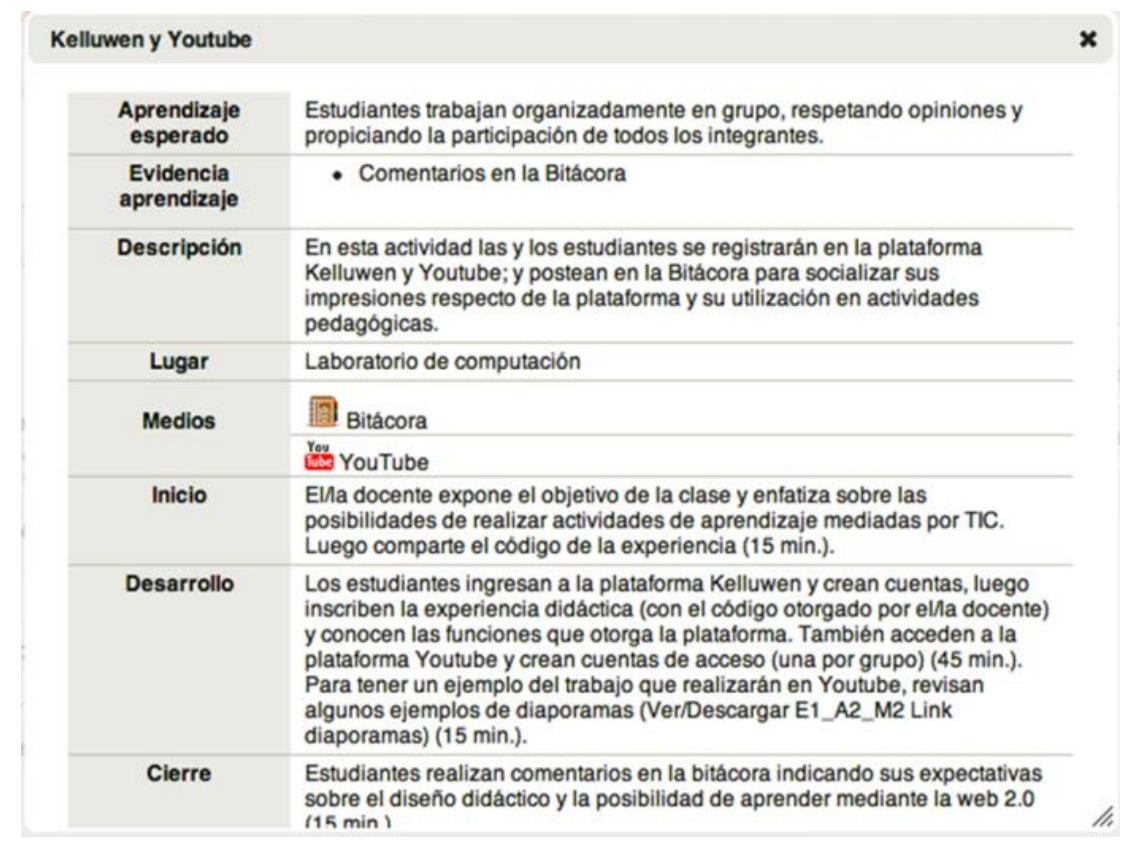

\subsection{LA ARTICULACIÓN GLOBAL DE LA EXPERIENCIA DIDÁCTICA EN KELLUWEN}

Llamamos Experiencia Didáctica a un DDC inscrito por un profesor y ejecutado en un aula, por lo tanto, las experiencias didácticas para un mismo DDC tendrán particularidades de ejecución en cada aula y serán enriquecidas con las interacciones educomunicativas de profesores y estudiantes. En lo sucesivo, hablaremos de Experiencia Didáctica para referirnos en conjunto al DDC y la experiencia particular que se desprende de la ejecución del DDC en aula.

En la Experiencia Didáctica, siguiendo el lineamiento del DDC correspondiente, se distinguen tres grandes etapas: Motivación, Creación y Evaluación. Cada etapa consiste en un conjunto de actividades (sesiones) que fomentan procesos dialógicos, reflexivos y progresivos de aprendizaje (ver Figuras 2 y 3 ).

La Etapa de Motivación: Esta primera etapa implica la socialización de experiencias previas de los estudiantes respecto de los objetivos curriculares y transversales a desarrollar. Contiene la presentación del Diseño Didáctico y la metodología de trabajo, además de ejemplificar las evidencias de aprendizaje que se esperan conseguir al final del proceso de innovación didáctica. La motivación se realiza en 1 a 3 sesiones en las cuales el profesor/a da a conocer a los estudiantes características generales del DDC, como la dinámica de grupos colaborativos, la organización del proceso de enseñanza - aprendizaje en tres etapas (Motivación, Creación, Evaluación) y la incorporación de herramientas de la Web 2.0. El docente como un guía y colaborador de los procesos de aprendizaje recupera experiencias previas y presenta los contenidos a partir de los contextos desde los que cada estudiante coopera y participa. Los roles de los estudiantes son definidos y sugeridos en el DDC de acuerdo a las características propias del diseño, sus contenidos y el uso de las herramientas. Por ejemplo, en un DDC centrado en la realización de una revista de historia, los roles pueden ser: historiador, editor y diseñador. 
La Etapa de Creación: Esta etapa, con 4 a 7 sesiones en aula y laboratorio de computación, está destinada a la elaboración de evidencias de aprendizaje que serán publicadas en la Web. Aquí se articula la inserción de los contenidos curriculares que cada diseño en particular busca cubrir, y el desafío de construir una pedagogía activa apoyada por la Web Social. Los estudiantes, trabajando en grupos y de acuerdo a lo roles asignados en la primera etapa, se embarcan en un proceso de investigación y construcción colaborativa de conocimientos sobre temáticas o contenidos que están presentes en el currículo escolar para la elaboración de evidencias de aprendizajes que toman forma de mensajes audio-escrito-visuales (Cebrian, 2009; Cloutier, 2001) que quedan disponibles en la Web. Un elemento motivacional importante en esta fase es que los y las estudiantes saben que estas evidencias o avances serán auto y co-evaluados por otros estudiantes, de la propia aula y de otras aulas gemelas, en actividades especialmente diseñadas para promover la revisión crítica y constructiva de los trabajos.

Las primeras sesiones están destinadas a extraer y recopilar información desde la web, libros, cartas, biografías, realización de entrevistas, entre otros. Toda esta exploración de medios y actividades son propuestas en forma ordenada mediante guías y pautas de trabajo disponibles en el módulo de gestión de avance de la plataforma Kelluwen, que orientan e intencionan la búsqueda según el propósito didáctico y curricular de cada diseño y la herramienta Web 2.0 asociada.

Al finalizar la etapa de Creación cada grupo habrá construido su trabajo, evidencia de aprendizaje que es síntesis de la investigación realizada, a través del uso de procesadores de texto, edición de imágenes, música, sonido, video y herramientas de presentación. Luego, estos trabajos son compartidos con las aulas gemelas, mediante su publicación en la herramienta Web utilizada (Youtube, Slideshare, Panoramio, GigaPan, etc.), y a la gestión inteligente aportada por la herramienta de Trabajos de la plataforma Kelluwen (ver Figura 4).

Figura 4. Publicaciones en la Herramienta de Trabajos de la plataforma Kelluwen

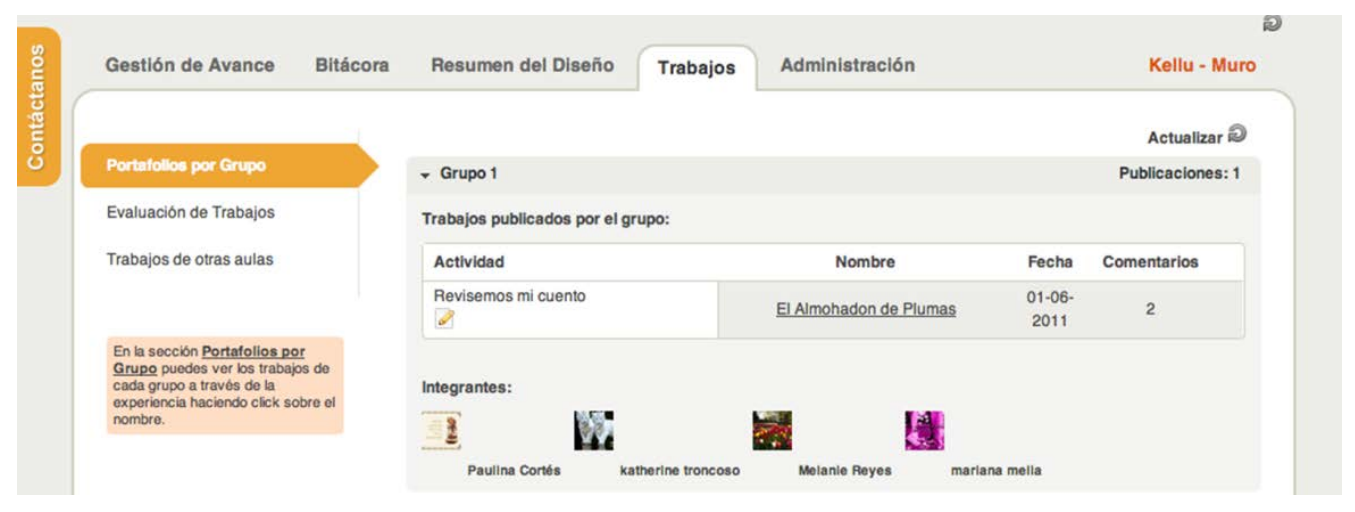

La Etapa de Evaluación: En esta etapa se retoman los objetivos curriculares socializando los niveles de logro a través de la evaluación de evidencias de aprendizaje en el aula y entre aulas gemelas. Además, se busca realizar un barrido desde los conocimientos previos hasta la construcción de nuevos conocimientos propuestos en el aula propia y en las gemelas. Los grupos de estudiantes valoran y evalúan las evidencias de aprendizaje de otros dos grupos asignados por el módulo de Trabajos de la plataforma Kelluwen. La interacción 
entre aulas gemelas permite la visualización de diversas evidencias de aprendizajes, lo que genera una gran posibilidad para que los estudiantes conozcan más de un enfoque u opinión respecto a un mismo objeto de estudio. Mediante este ejercicio los estudiantes reconocen que se puede acceder a distintas opiniones y formas de generar modos explicativos variados para un mismo contenido. Los estudiantes relacionan sus aprendizajes con los trabajos que evalúan estableciendo puntos de comparación en la medida que las diferencias entre una evidencia y otra posibilitan la retroalimentación de los contenidos curriculares. Además, se facilita una instancia para realizar opiniones críticas basadas en los soportes audio-escritovisuales o escrito-visuales construidos por cada grupo de trabajo y el respeto a las creaciones del otro. En efecto, a través de un algoritmo de asignación de pares revisores, a los grupos de las distintas aulas gemelas se les asignan dos trabajos para ser co-evaluados a través de una pauta disponible en la plataforma Kelluwen. En la Figura 5 se puede apreciar el despliegue de la asignación de trabajos a evaluar y de las evaluaciones recibidas por cada grupo.

La Experiencia Didáctica finaliza con una autoevaluación grupal o individual orientada hacia la reflexión sobre los procesos meta-cognitivos desarrollados durante el aprendizaje.

Figura 5. Etapa de evaluación en Herramienta de Trabajos de plataforma Kelluwen

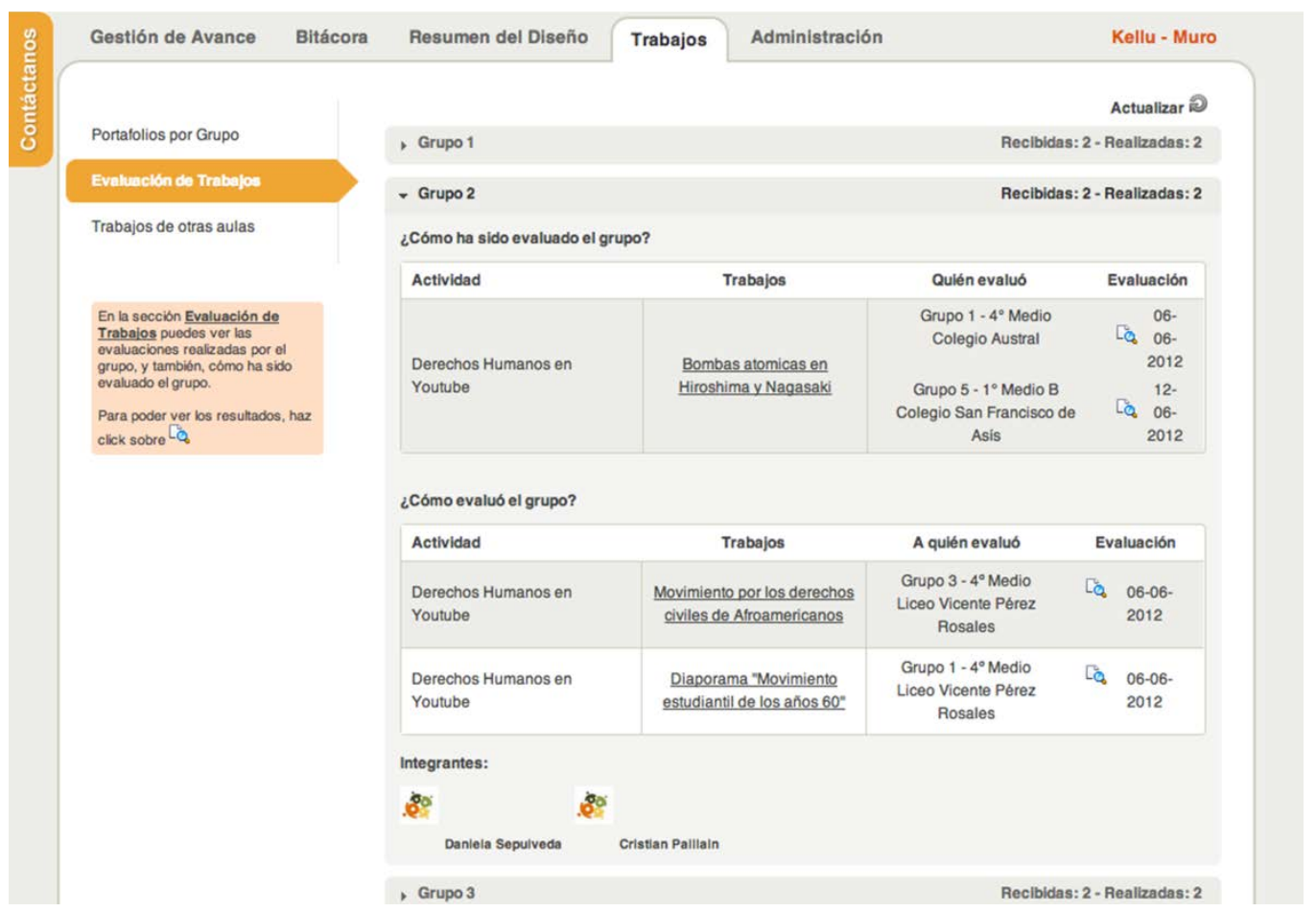

\section{RESULTADOS DE TRES AÑOS DE EXPERIENCIAS KELLUWEN}

A continuación, se presentan los resultados de varios estudios. Se analiza el impacto del proyecto en la adquisición de competencias socio-comunicativas, las características de 
los profesores respecto de sus niveles de participación, la conformación de la red social Kelluwen y la percepción de estudiantes frente al proceso.

\subsection{IMPACTO EN LAS COMPETENCIAS SOCIO-COMUNICATIVAS}

El análisis de la variación de habilidades socio-comunicativas se realiza a partir de un pre y post test denominado "Diagnóstico de competencias socio-comunicativas", aplicado a un total de 79 estudiantes de $7^{\circ}$ nivel de enseñanza básica en cuatro aulas de escuelas de la ciudad de Valdivia. Tres de estas aulas (57 estudiantes) ejecutaron un Diseño Didáctico Kelluwen. La cuarta aula (22 estudiantes) es control. Para medir las habilidades de lecto-escritura de los alumnos, se emplea la técnica Test Cloze (Condemarín y Milicic, 1988). Se construyeron dos versiones del test con dificultades similares y se aplicaron aleatoriamente en las fases pre y post, con el propósito de eliminar el factor de diferencia entre ambas versiones en el análisis de datos. Los resultados de los tests fueron evaluados por dos jueces independientes.

Variación de competencia socio-comunicativa: Producción de textos. Con el fin de evaluar las competencias asociadas a la capacidad producir textos, se consideró la redacción de un texto narrativo. Las rúbricas contienen los criterios de: ortografía, redacción, contenido, originalidad, vocabulario, cohesión y coherencia, énfasis en ideas relevantes y estructura genérica narrativa.

Para evaluar si existe un aumento del nivel alcanzado en cada criterio entre pre y post test, se calcula una tabla de contingencia pre-post considerando las distribuciones empíricas condicionales a la categoría obtenida en la rúbrica por cada estudiante en el pretest, de manera de poder visualizar los eventuales progresos de categoría en el post-test. Esta información se acompaña con un resumen de los efectivos sobre, bajo y en la diagonal de la tabla de contingencia. Luego, se calcula el estadístico de Bowker (Bowker, 1948) para el test de simetría en muestras dependientes de variables categóricas. Este cálculo se presenta en la Figura 6 (ver Agresti, 1996).

Figura 6. Estadístico de Bowker para test de simetría de muestras dependientes

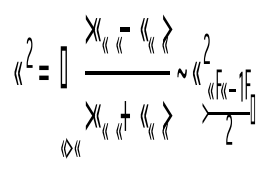

Si no se rechaza la hipótesis de simetría, significa que no hay diferencias significativas entre pre y post test. Si se rechaza la hipótesis de simetría, y además el número de efectivos sobre la diagonal es mayor que aquellos bajo la diagonal, entonces se puede concluir que ha habido un aumento significativo del nivel del criterio en análisis entre el pre y post test.

Aplicando esta metodología, el único criterio que presenta una diferencia significativa entre pre y post test en los grupos de estudio es el criterio cohesión en la redacción. La rúbrica de este criterio se presenta en la Tabla 1. 
Tabla 1. Rúbrica asociada al criterio de cohesión en la redacción

\begin{tabular}{|c|c|c|c|}
\hline $\begin{array}{c}\text { Totalmente Logrado } \\
\text { (4) }\end{array}$ & $\begin{array}{c}\text { Logrado } \\
\mathbf{( 3 )}\end{array}$ & $\begin{array}{c}\text { Poco Logrado } \\
\mathbf{( 2 )}\end{array}$ & $\begin{array}{c}\text { No Logrado } \\
\text { (1) }\end{array}$ \\
\hline $\begin{array}{c}\text { El estudiante realiza un } \\
\text { uso correcto de } \\
\text { conectores a lo largo del } \\
\text { texto. }\end{array}$ & $\begin{array}{c}\text { Usa conectores a lo largo } \\
\text { del texto, ayudando a la }\end{array}$ & $\begin{array}{c}\text { El uso de conectores es } \\
\text { comprensión del mismo. impidiendo una } \\
\text { cuena comprensión del } \\
\text { texto. }\end{array}$ & $\begin{array}{c}\text { No hay uso de } \\
\text { conectores, lo que } \\
\text { difículta la } \\
\text { comprensión del texto. }\end{array}$ \\
\hline
\end{tabular}

En la Figura 7 se presentan los resultados de la evaluación del Criterio Cohesión en la Redacción, correspondientes a los 20 estudiantes del aula control.

Figura 7. Tabla de contingencia evaluación pre-post test Criterio Cohesión en Grupo Control

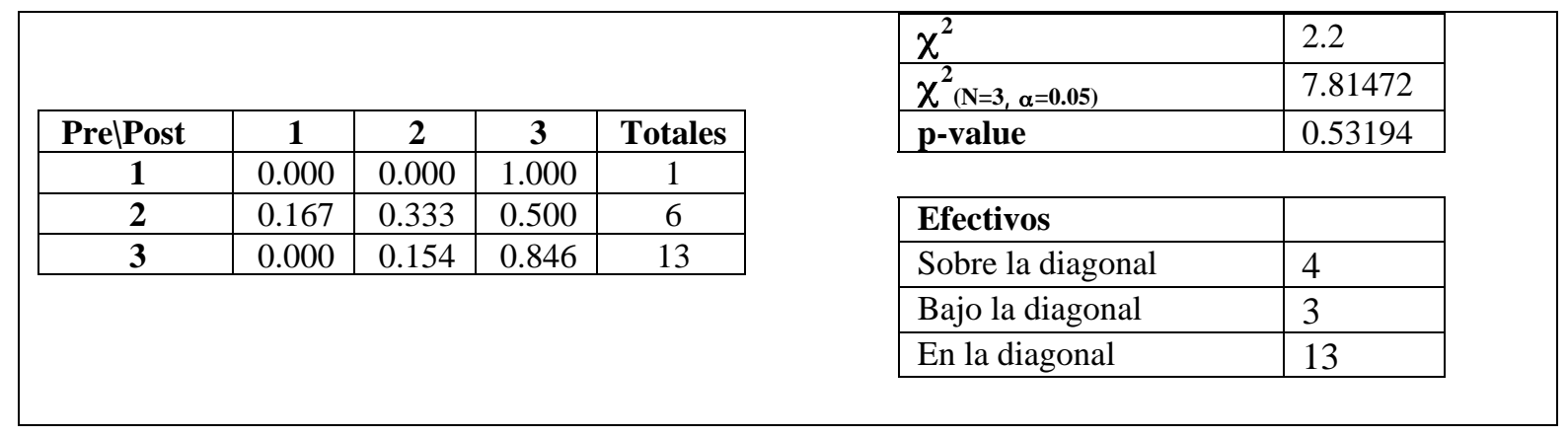

En este caso, el test de Bowker nos indica que no se rechaza la hipótesis de simetría al 95\% de confianza: no hay una diferencia significativa entre los resultados de pre y posttest en el caso control.

En la Figura 8 se presentan los resultados de la evaluación del Criterio Cohesión en la Redacción, correspondientes a los 54 estudiantes de las 3 aulas en estudio. En este caso se rechaza el test de simetría al 95\% de confianza, y se observan 36 casos efectivos sobre la diagonal versus 10 bajo la diagonal. Esto nos permite concluir que hay un aumento significativo del nivel de este criterio en los estudiantes que ejecutaron la experiencia didáctica Kelluwen.

Figura 8. Tabla de contingencia evaluación pre-post test Criterio Cohesión en Grupo en Estudio

\begin{tabular}{|c|c|c|c|c|c|}
\hline PrelPost & $\mathbf{1}$ & $\mathbf{2}$ & $\mathbf{3}$ & $\mathbf{4}$ & Total \\
\hline $\mathbf{1}$ & 0.000 & 0.285 & 0.430 & 0.285 & 7 \\
\hline $\mathbf{2}$ & 0.143 & 0.143 & 0.214 & 0.500 & 14 \\
\hline $\mathbf{3}$ & 0.094 & 0.125 & 0.188 & 0.593 & 32 \\
\hline $\mathbf{4}$ & 0.500 & 0.000 & 0.000 & 0.500 & 2 \\
\hline
\end{tabular}

\begin{tabular}{|l|l|}
\hline $\boldsymbol{\chi}^{\mathbf{2}}$ & 26.4761 \\
\hline $\boldsymbol{\chi}_{(\mathrm{N}=\mathbf{3}, \boldsymbol{\alpha}=\mathbf{0 . 0 5 )}}$ & 12.5915 \\
\hline $\mathbf{p}$-value & 0.00018 \\
\hline
\end{tabular}

\begin{tabular}{|l|c|}
\hline Efectivos & \\
\hline Sobre la diagonal & 36 \\
\hline Bajo la diagonal & 10 \\
\hline En la diagonal & 9 \\
\hline
\end{tabular}


Variación de competencia socio-comunicativa: Habilidad lecto-escritural. Un aspecto relevante para el estudio que se presenta, y cuya variación destaca por sobre el resto, es la habilidad lecto-escritural observada en los estudiantes de las aulas que desarrollaron la experiencia didáctica Kelluwen. Siguiendo la técnica Cloze, los niveles de lecto-escritura corresponden a un porcentaje de logro y se midieron con un ejercicio que implicó otorgar sentido a la estructura genérica narrativa de un texto por medio de la elección y redacción de palabras. El índice es, finalmente, clasificado en tres niveles de lectura: nivel de frustración (0\% a 43\%), nivel instruccional (44\% a $74 \%$ ) y nivel independiente (75\% a 100\%). El nivel independiente significa que el alumno lee con fluidez, precisión y comprende la mayor parte del texto. En el nivel instruccional la lectura es medianamente fluida, con algunas dificultades en el reconocimiento de palabras y, aunque el lector capta el contenido y su estructura, evidencia algunas fallas en la comprensión. Por último el nivel de frustración implica que son numerosos los errores de reconocimiento de palabras, y que la comprensión es ciertamente deficiente.

En la Figura 9 (primera fila) se presentan histogramas del índice Cloze obtenido por 22 estudiantes del aula control en el pre y post-test, además de la diferencia individual entre pre y post test. Este último histograma nos revela que alrededor del $50 \%$ de estos estudiantes mantuvo valores del índice Cloze muy similares entre pre y post-test. Más aún, la hipótesis de que la media de las diferencias es cero no se rechaza con un 95\% de confianza (ver Tabla 2). Esto significa que en el aula control no hay diferencias significativas en las habilidades lecto-escritural entre pre y post-test.

Figura 9. Histogramas Índice Cloze en el Aula Control y en las Aulas en Estudio
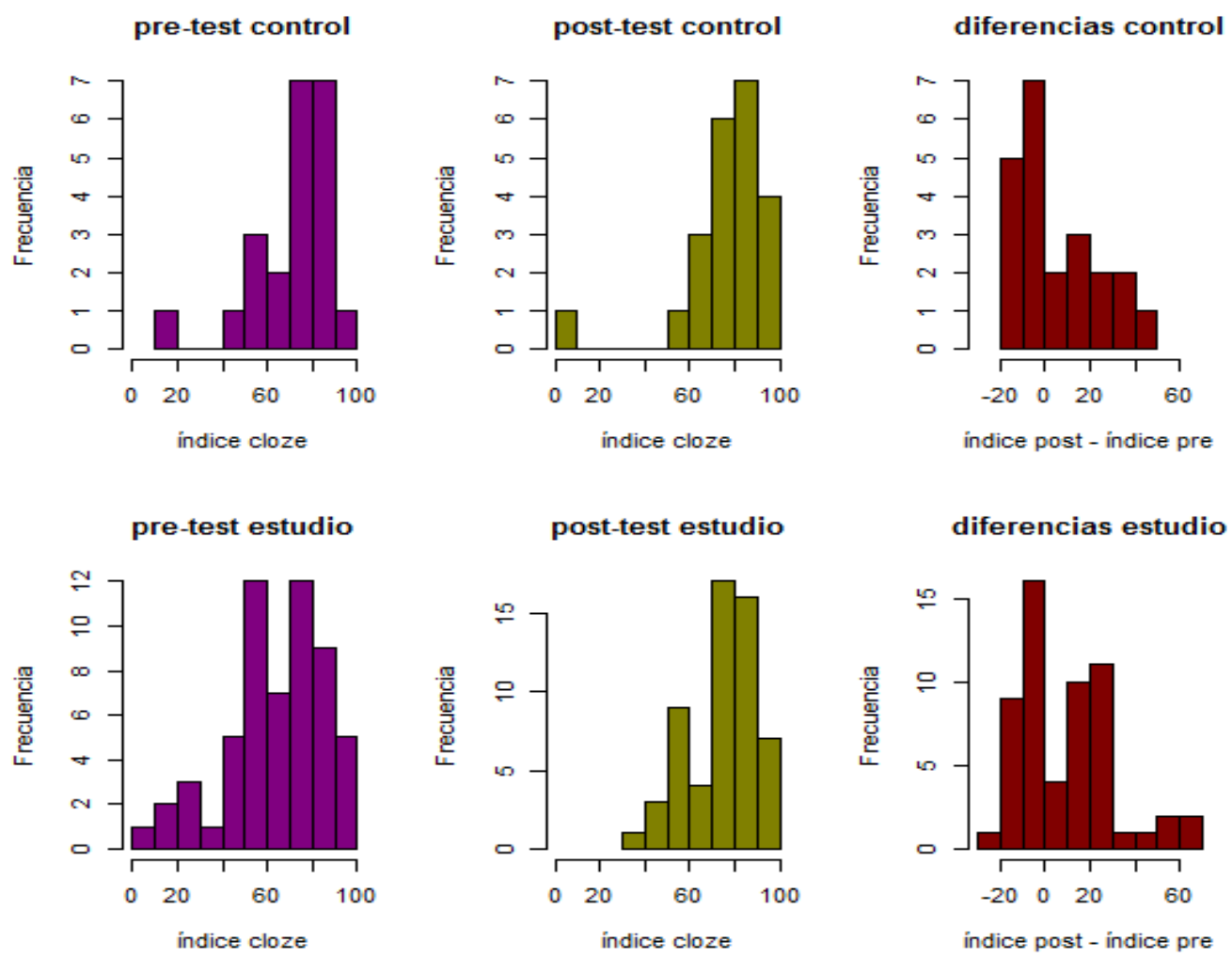
Estudios Pedagógicos XXXIX, Número Especial 1: 111-131, 2013

Tabla 2. Resultados análisis estadístico de la variación del índice Cloze entre pre y post test en Aula Control y Aulas en Estudio

\begin{tabular}{|l|l|l|l|l|}
\hline Población & T Muestral & $\begin{array}{l}\text { Grados lib. T- } \\
\text { student }(\mathbf{d f})\end{array}$ & T(df, 0,975) & Interpretación \\
\hline Aula Control & 1.06787 & 21 & 2.079614 & $\begin{array}{l}\text { Diferencias no } \\
\text { significativas }\end{array}$ \\
\hline Aulas en Estudio & 3.983696 & 56 & 2.003241 & $\begin{array}{l}\text { Diferencias } \\
\text { significativas }\end{array}$ \\
\hline
\end{tabular}

En la misma Figura 9, segunda fila, se presentan histogramas del índice Cloze obtenidos en el pre y post-test por 57 estudiantes de las aulas que desarrollaron la experiencia didáctica Kelluwen, junto a la diferencia individual entre pre y post test. Este último histograma muestra que más del $50 \%$ de los estudiantes aumentó el valor del índice Cloze entre pre y post-test. Más aún, la hipótesis de que la media de las diferencias es cero se rechaza con un $95 \%$ de confianza (ver Tabla 2). Lo anterior evidencia que en este caso sí hay un aumento significativo del índice Cloze entre pre y post-test. Otro aspecto importante que se observa en este caso, es que alrededor del 59\% de los estudiantes alcanza el nivel de independiente de lecto-escritura en los resultados del post-test, frente al $37 \%$ de estudiantes de este nivel en el pre-test.

\subsection{CARACTERIZACIÓN DE LOS PROFESORES FRENTE A LA INNOVACIÓN}

Utilizando datos de la actividad en la plataforma Kelluwen de los profesores que desarrollaron experiencias didácticas durante el año 2011, se realiza un estudio que busca caracterizar los distintos modos en que los profesores se involucran en la dinámica de innovación. En este estudio se incluyen los profesores que avanzaron al menos un $20 \%$ en la experiencia didáctica propuesta, que corresponde a 55 maestros de un total de 68 profesores inscritos en la red Kelluwen. Se identificaron nueve variables que están relacionados con las principales actividades de interacción de los profesores en la plataforma. Una primera variable es el número de diseños didácticos desarrollados por cada docente, llamado $\mathrm{N}^{\mathrm{o}}$ DDC en el análisis. A partir del módulo de Gestión de Avance se calcula la media y desviación estándar del tiempo entre el inicio de dos actividades consecutivas del diseño didáctico ejecutado por el maestro, variables que denominamos Tiempo medio y Desviación Estándar (DE) de Tiempo respectivamente. En el módulo Bitácora, el profesor interactúa con sus alumnos durante las sesiones en laboratorio de computación, lo cual es representado con las variables Número de Mensajes, Número de Respuesta y Número de Valoraciones que emite el profesor en la Bitácora durante todo el desarrollo de sus experiencias didácticas. En el módulo denominado Kellu-Muro, los profesores interactúan con sus colegas que desarrollan el mismo diseño didáctico, lo cual es representado con las variables Número de Mensajes, Número de Respuesta y Número de Valoraciones que emite el profesor en el Kellu-Muro.

Con estas variables definidas, se desarrolla un análisis de agrupamiento o clustering para descubrir patrones de comportamiento de los profesores en su interacción con la 
plataforma Kelluwen. Se trata de descubrir grupos de profesores con patrones de interacción similares. Para ello se utilizan dos técnicas de agrupamiento: K-means (Hartigan y Wong, 1979) y Análisis de Clases Latentes (LCA) (Magidson y Vermunt, 2004), obteniéndose con ambos resultados muy similares (Scheihing et al., 2012). En la Figura 10 se muestran los grupos obtenidos con la técnica $\mathrm{K}$-means para $\mathrm{K}=5$, proyectados en el plano de componentes principales obtenidos de los datos. Se observa que hay un grupo principal con 43 individuos que no presenta características distintivas especiales. Sin embargo, se observa cuatro grupos minoritarios con un patrón de comportamiento bien definido. Dos de ellos corresponden a grupos de un solo individuo que se caracterizan por un uso intensivo de la Bitácora. El tercero de ellos es el grupo 3 con 5 individuos que se caracterizan por tener tiempos promedio entre el inicio de actividades consecutivas mucho mayores que la mayoría de los profesores, junto a una dispersión mayor en estos mismos tiempos. El cuarto es el grupo 2 con 3 individuos que se caracterizan por usar los servicios de Web Social (Bitácora y Kellu-muro) de manera más intensiva que la mayoría de los profesores.

Una observación importante que concierne a los dos últimos grupos descritos, es que la variable Tiempo entre actividades y las variable relacionadas con el uso de servicios de Web Social de la plataforma Kelluwen, aparecen en ejes ortogonales en la proyección de los datos en el plano de componentes principales. Este hallazgo sugiere que aquellos profesores que usan más frecuentemente los servicios de Web Social de la plataforma Kelluwen presentan un desarrollo más regular de sus experiencias didácticas.

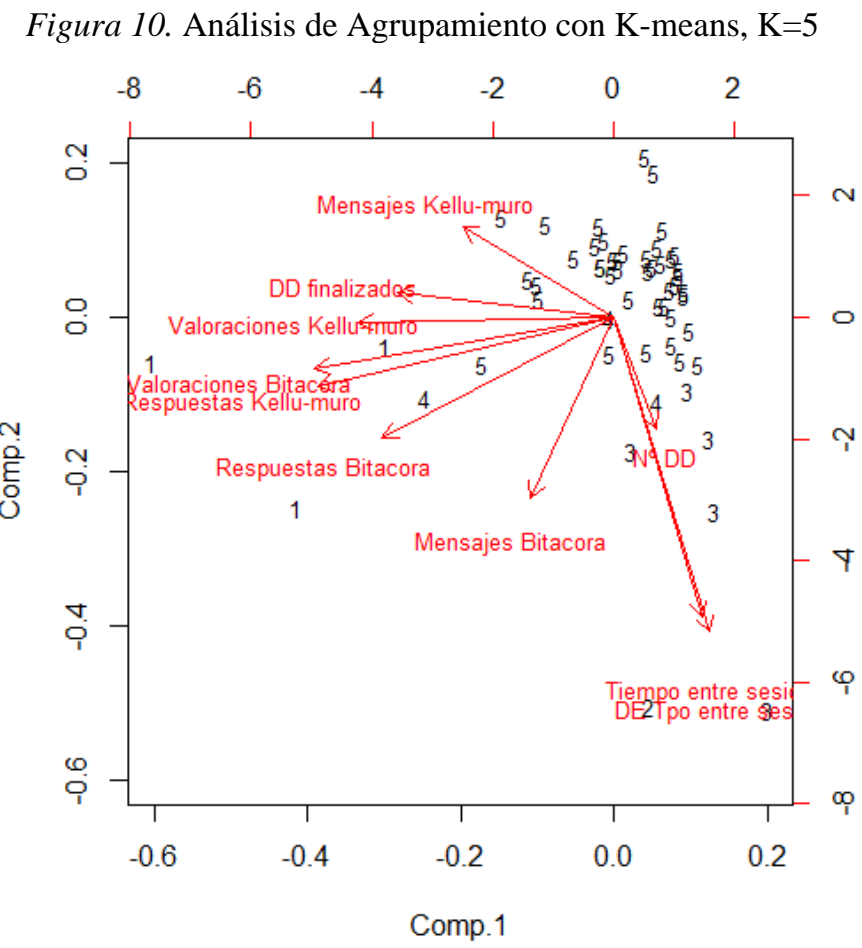

\subsection{LA RED SOCIAL KELLUWEN}

Con el objeto de estudiar el efecto de la innovación didáctica propuesta por 
Kelluwen en la formación de la comunidad de aprendizaje Kelluwen, se desarrollan diversos análisis de redes sociales para las interacciones de estudiantes y profesores en la plataforma Kelluwen.

La Bitácora es el módulo que registra el mayor número de interacciones de estudiantes y profesores en la red Kelluwen. Se considera como interacciones las respuestas o valoraciones a los mensajes que estudiantes y profesores escriben en la Bitácora. Un total de 5355 interacciones se registraron en la red durante el primer semestre del 2011, correspondientes a 1400 usuarios (estudiantes y profesores). Estos datos nos permiten generar un grafo de interacciones, para el cual se han calculado dos medidas de centralidad clásicas: la centralidad de vector propio, que mide la importancia de un nodo del grafo y la intermediación que mide la característica de que un nodo sea enlace entre otros nodos (ver Hanneman y Riddle, 2012). Para cada medida, se seleccionan los 6 usuarios (nodos) con los valores más altos quienes se identifican explícitamente en el grafo (ver Inalef et al., 2012). La Figura 11 muestra el grafo de interacciones en el cual se han destacado los actores con los más altos valores de la medida de intermediación. Al asociar los identificadores con los datos de cada usuario se constata que los principales actores son 3 profesores y 3 asistentes del equipo de investigación que trabajaron acompañando a los profesores en el desarrollo de los diseños didácticos. Cabe notar que el grafo resultante se caracteriza por una estructura de interacciones locales que representan la actividad al interior de cada aula en el desarrollo de sus experiencias didácticas, y con una menor frecuencia de interacciones entre aulas en las que profesores e investigadores aparecen como actores claves.

Figura 11. Grafo de interacciones en la Bitácora en donde los nodos con mayores valores de la medida de intermediación han sido etiquetados

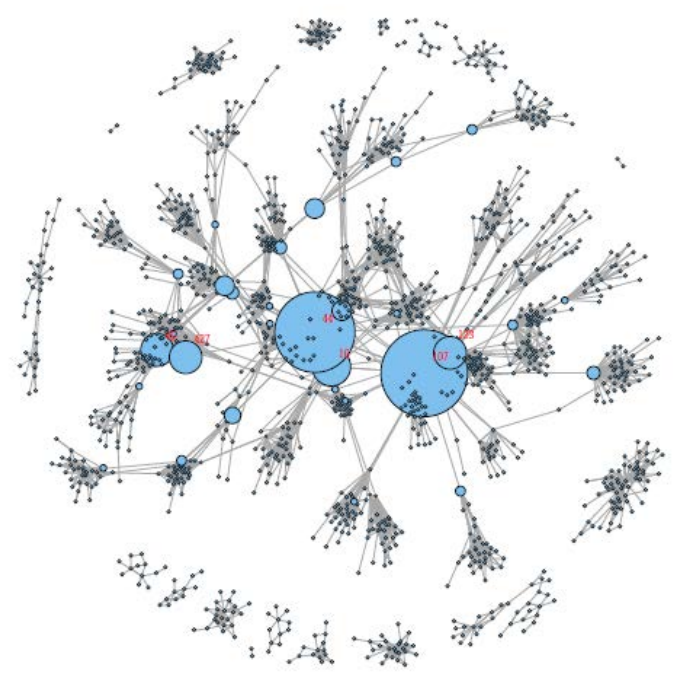

\subsection{ESTUDIO CUALITATIVO DEL PROCESO DE INNOVACIÓN}

Con el objetivo de relacionar el discurso de los estudiantes frente al escenario educacional innovador, se realiza un análisis cualitativo a partir de dos grupos focales realizados a un total de 16 estudiantes mujeres en dos aulas (aulas A y B con 8 estudiantes 
cada una) de $7^{\circ}$ Básico en una de las escuelas en estudio (Flores, 2012). Los textos transcritos se analizan identificando códigos significativos y se construyen mapas de sentido discursivo, utilizando el software QDA Miner ${ }^{1}$. Si bien el entorno educativo de ambos grupos es similar (misma escuela, mismo nivel), cada aula aplicó la experiencia pedagógica guiada por docentes con distinto perfil motivacional. Cabe entonces realizar el análisis de discurso de cada grupo por separado para identificar diferencias y semejanzas presentes en las motivaciones y percepciones entre ambas aulas tras la ejecución del DD.

Los resultados del análisis, que se presentan en detalle en Flores (2012), dan cuenta de diferencias entre las aulas en el uso conceptos relevantes a la innovación educativa, y relacionan estas diferencias con características motivacionales y de competencia de las respectivas profesoras. Para resumir los resultados, se presenta primero un análisis de las frecuencias de conceptos clave, y luego un análisis de la relación o interactividad de éstos.

\subsubsection{FRECUENCIA DE CONCEPTOS CLAVES}

En ambas aulas, se constata que las estudiantes otorgan especial énfasis a un gran número de conceptos que presentan niveles similares de frecuencia dentro del discurso. La alta frecuencia de los conceptos "utilización de las TIC en el contexto escolar", "motivación TIC- Web 2.0" y "aprendizaje significativo" da cuenta de la concepción del contexto tecnológico como una potencial oportunidad de aprender a través de la colaboración. Según lo expresado por las estudiantes, el contexto y las herramientas ofrecidas por la experiencia didáctica les permitió aprender e integrar conocimientos que del modo tradicional -o automatizado- no lograron interiorizar.

Además, se encuentra una clara diferencia entre ambas aulas respecto de los conceptos asociados a la relación con la docente. En efecto, en el discurso de las estudiantes del aula A se evidencia la presencia de los conceptos "valoración positiva" y "género femenino", en cambio en el aula B, dichos conceptos no estuvieron presentes. Las estudiantes del aula A destacan la influencia que cobra para ellas la presencia de la profesora en las actividades pedagógicas Kelluwen, mientras que las estudiantes del aula B refieren nulo impacto al rol de la docente.

\subsubsection{CLUSTERS: EFECTOS DE INTERACTIVIDAD DE LOS CÓDIGOS}

El análisis de este apartado hace referencia a los conceptos centrales que presentaron una asociación espontánea, producto de la relación y frecuencia de su interacción durante el discurso de las alumnas de cada aula. Para cada curso analizado se presenta el cluster o familias de códigos de mayor significación.

Cluster $N^{\circ} 1$ Aula A: Motivaciones por una educación constructivista. En el aula A se observa la asociación de códigos ligados al proceso de enseñanza-aprendizaje, motivación, rasgos constructivistas de la adquisición del conocimiento y la influencia que posee la inclusión de las TIC en dicho contexto. Este agrupamiento se muestra en la Figura 12 como "Familia 1". En esta familia, la motivación asociada al constructivismo se vincula a una mayor a libertad de acción en sus aulas, a las ganas de interactuar con sus pares tanto dentro como fuera de la escuela, a la noción de entretenimiento y mayor apropiación sobre

\footnotetext{
${ }^{1}$ http://provalisresearch.com/products/qualitative-data-analysis-software/qda-miner-benefits/
} 
los contenidos a aprender, situación que según el análisis se transforma en un modo dinámico y entretenido de aplicar los contenidos curriculares. El protagonismo, descubrimiento y significación son factores que se conjugan y se vinculan con los conceptos motivacionales propios del contexto pedagógico en el que Kelluwen intervino. En otras palabras, la utilización de las herramientas tecnológicas permitió a las estudiantes tomar mayor protagonismo en la construcción de conocimientos propios, dejando atrás la característica pasividad de las clases conductistas o instruccionales a las que están acostumbradas.

Figura 12. Cluster N ${ }^{\circ}$, Aula A: "Motivación, TIC y constructivismo"

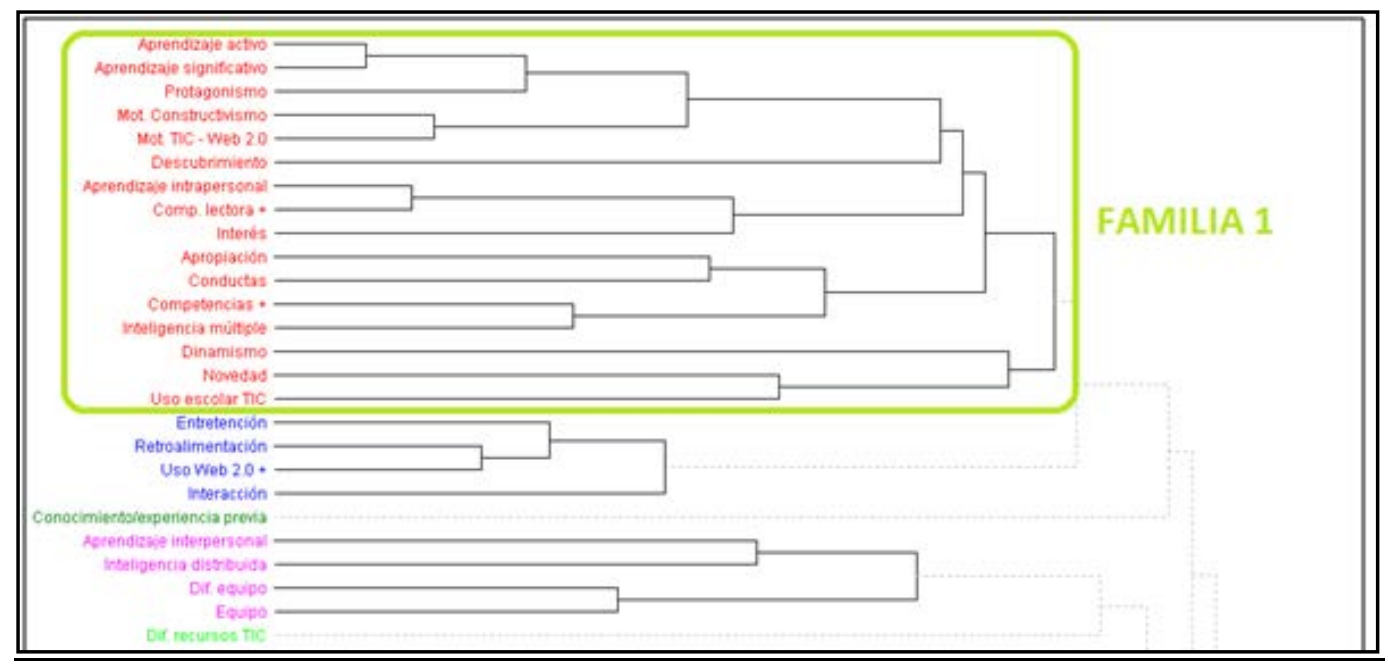

Cluster $\mathrm{N}^{\mathrm{o}} 1$ Aula B: Aprendizaje y protagonismo. En el aula B se observa un primer nexo significativo entre los conceptos de descubrimiento y aprendizaje activo, donde las estudiantes ven una oportunidad para dejar la inmovilidad a un lado y dar paso a una mayor participación en sus caminos como aprendices (ver Familia 1 en Figura 13).

La sensación de protagonismo en la escuela -otorgado por los rasgos constructivistas de la metodología Kelluwen- se sitúa como un factor de alta motivación, debido al dinamismo que incorpora a las rutinas diarias de los establecimientos. La opción de descubrir y aplicar la teoría a actos concretos como la indagación sobre contenidos específicos por medio de las TIC, sitúa a las estudiantes en un escenario nuevo y atractivo. Sin embargo, se constata una clara disminución en la cantidad de agrupamientos y conceptos vinculados en el aula $\mathrm{B}$ con respecto al aula $\mathrm{A}$.

Figura 13. Cluster $\mathrm{N}^{\mathrm{o}}$ 1, Aula B: Aprendizaje y protagonismo

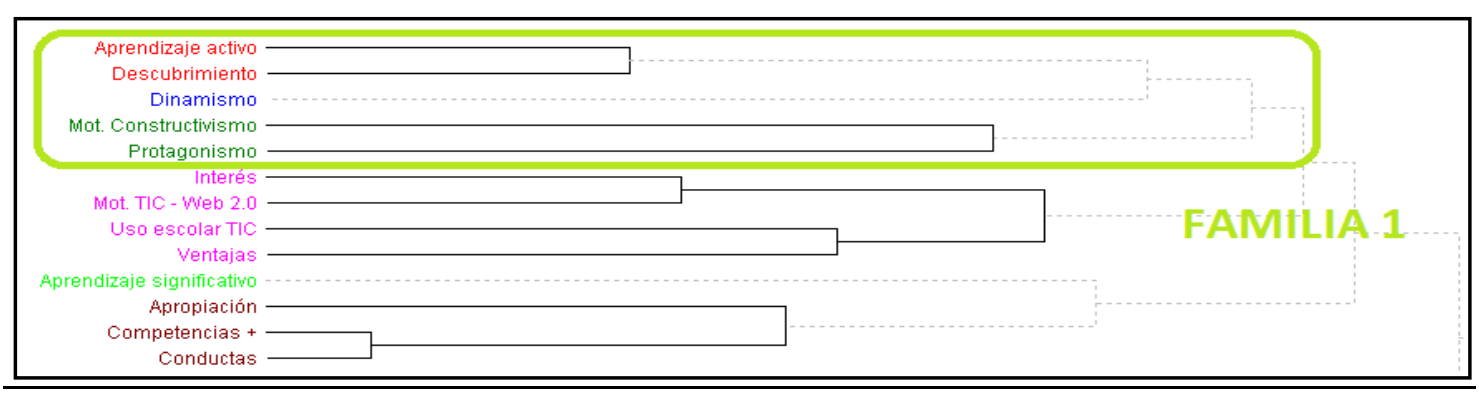


Tras el análisis realizado, podemos deducir que los factores que presuponen ser el motivo de las diferencias cualitativas plasmadas en el estudio fueron: la valoración positiva hacia la docente, tanto por el sentimiento de confianza generado tras Kelluwen, como por sus competencias TIC; el sentimiento de colaboración y apoyo mutuo para conseguir un objetivo común, evidenciado tanto por la docente del aula A como por sus alumnas; la asimilación de las herramientas TIC no sólo como un espacio/medio de entretención, sino como recursos eficientes y útiles para conseguir objetivos de aprendizaje escolar; la asociación de aprendizaje significativo a la posibilidad de búsqueda, protagonismo y descubrimiento, esto basado en las pautas conductuales cotidianas de las estudiantes en el universo de los recursos e interacción Web 2.0; la reflexión acerca de la desmotivación provocada por los factores ligados a dinámicas conductistas, tales como la pasividad, jerarquía, individualismo y automatización.

\section{CONCLUSIONES}

Tres años implementando la metodología Didáctica 2.0 y conociendo muy de cerca diversos contextos escolares de la zona sur austral, nos han permitido concluir que los elementos claves de la innovación con TIC son la formación de los profesores, su apropiación de las propuestas, el acompañamiento en aula con el objetivo de generar niveles graduales de autonomía en el uso de TIC y el abordaje de la innovación, junto al reconocimiento de ésta por parte de todos los actores de la comunidad escolar. Los resultados cualitativos dan cuenta de la respuesta de los estudiantes en cuanto a la apropiación y protagonismo en el proceso de aprendizaje, así como también de aspectos motivacionales que posibilitan mejores experiencias educativas. Los resultados muestran también claras diferencias entre aulas con docentes de distinto perfil motivacional y de habilidades tecnológicas. El efecto positivo en los estudiantes es claramente mayor cuando el docente está comprometido y tiene un perfil de habilidades tecnológicas adecuado. Esto reafirma la idea de que aunque el proceso de aprendizaje se centra en el estudiante, el rol del maestro es fundamental como mediador y facilitador. Lo que hemos aprendido en Kelluwen refuerza la idea de que para conseguir innovaciones educativas con permanencia en el tiempo, las propuestas deben considerar intervenciones de manera más integral, es decir, articulando todo el centro escolar y no los cursos, los profesores o los estudiantes de manera aislada. En este sentido, la metodología de desarrollo en espiral, que busca mejorar las propuestas de innovación con la retroalimentación de los propios actores del proceso, nos ha permitido generar intervenciones contextualizadas y pertinentes que han sido positivamente valoradas por los profesores y estudiantes.

Ciertamente, el mejoramiento de las capacidades lecto-escritoras de nuestros/as estudiantes requiere de un proceso re-formativo extenso, pues se trata de modificar las estructuras metodológicas de la educación chilena. Intervenciones como las propuestas por Kelluwen, que apuestan por poner la acción en manos del estudiante, por acercar los contenidos al contexto sociocultural inmediato de los mismos, por dar la posibilidad de escribir, leer, releer, desmenuzar y analizar los textos, son un acercamiento para producir efectos positivos en las habilidades asociadas a la lecto-escritura. Los resultados obtenidos en el proyecto son promisorios, pues aunque el mejoramiento en los aprendizajes medidos 
Estudios Pedagógicos XXXIX, Número Especial 1: 111-131, 2013 LA EXPERIENCIA KELLUWEN: TRES AÑOS DE DESARROLLO Y PUESTA EN PRÁCTICA DE UNA PROPUESTA DE INNOVACIÓN DIDÁCTICA CON USO DE TIC

es modesto, las intervenciones en aula fueron de corta duración (1 -1,5 mes) y habría que considerar el efecto de aplicar esta metodología a largo plazo.

\section{REFERENCIAS BIBLIOGRÁFICAS}

Agresti, A. (1996). An introduction to categorical data analysis. New York: John Wiley \& Sons

Álvarez, I. (2009). Evaluar para contribuir a la autorregulación del aprendizaje. Electronic Journal of Research in Educational Psychology, vol.19, 1007-1030.

Arancibia, M. (2004). Una propuesta para trabajar en las escuelas con Internet: Gestión del conocimiento y comunidades de aprendizaje. Estudios Pedagógicos, n.30, 111-122.

Arancibia, M., Soto, C.P. y Contreras, P. (2010). Concepciones del profesor sobre el uso educativo de las TIC asociada a procesos de enseñanza-aprendizaje en el aula escolar. Estudios Pedagógicos, vol.36, n.1, 23-51.

Area, M. y Ribeiro, M.T. (2012). De lo sólido a lo líquido: las nuevas alfabetizaciones ante los cambios culturales de la Web 2.0. Comunicar, vol.XIX, n.38, 13-20.

Aubert, A., Flecha, A., García, C., Flecha, R. y Racionero, S. (2011). Aprendizaje Dialógico en la Sociedad de la Información. Barcelona: Editorial Hipatia.

Bilbao, A. y Salinas, A. (Ed.). (2010). El libro abierto de la informática educativa. Lecciones y desafíos de la Red Enlaces. Santiago: Enlaces, Centro de Educación y Tecnología del Ministerio de Educación.

Bowker, A. H. (1948). A test for symmetry in contingency tables. Journal of the american statistical association, vol.43, n.244, 572-574.

Cebrian, M. (2009). Comunicación interactiva en los cibermedios. Comunicar, vol.XVII, n.33, 1524.

Cloutier, J. (2001). Petit traité de communication. Emerec à l'heure des technologies numériques. Montréal: CarteBlanche

Coll, C. (2005). Concepciones y tendencias actuales en psicología de la Educación. En C. Coll, J. Palacios y A. Marchesi (Eds.), Desarrollo Psicológico y Educación. Vol. 2. Psicología de la educación escolar (pp. 29-66). Madrid: Alianza.

Condemarín, M. y Milicic, N. (1988). Test de cloze. Aplicaciones psicopedagógicas. Santiago: Andrés Bello.

Contreras, P., Arancibia, M. y Cárcamo, L. (2009). Hipermedios y cooperación: la Web 2.0 y su incidencia en los procesos educativos. En M. Segura y B. Onetto (Eds.), Diálogos Culturales II. Interfaces Viciadas, comunicación visual y otras mediaciones (pp. 177-221). San José de Rio Preto: Bluecom.

Flores, P. (2012). Aprendizaje mediado por tecnologías: Impacto de estrategias pedagógicas apoyadas por la Web 2.0 en estudiantes de la Escuela México Valdivia, Región de Los Ríos. Tesis de Magíster en Comunicación, Universidad Austral de Chile. Valdivia, Chile.

Hanneman, R. y M. Riddle. Introduction to Social Network Methods. Recuperado de http://faculty.ucr.edu/ hanneman/nettext/, visitado en enero del 2012.

Hargreaves, A. y Fullan, M. (2012). Professional Capital: transforming teaching in every school. New York: Teachers College Press, Columbia University

Hartigan, J. A. y Wong, M. A. (1979). Algorithm AS 136: A K-Means Clustering Algorithm. Journal of the Royal Statistical Society, Series C, vol.28, n.1, 100-108.

Hepp, P. (2003). Enlaces: el programa de informática educativa de la reforma educacional chilena. 
Estudios Pedagógicos XXXIX, Número Especial 1: 111-131, 2013 LA EXPERIENCIA KELLUWEN: TRES AÑOS DE DESARROLLO Y PUESTA EN PRÁCTICA DE UNA PROPUESTA DE INNOVACIÓN DIDÁCTICA CON USO DE TIC

En C. Cox (Ed.), Políticas educacionales en el cambio de siglo: La reforma del sistema escolar de Chile (pp. 419-451). Santiago: Editorial Universitaria.

Inalef, K., Guerra, J. y Scheihing, E. (2012). Development and Validation of a Virtual Worklog as a Collaboration Tool for Kelluwen Learning Community. Actas Third International Workshop on Interactive Environments and Emergent Technologies for eLearning, IEETel-2012, Liverpool-Inglaterra.

Kaplún, M. (1998). Pedagogía de la comunicación. Madrid: Ediciones de la Torre.

Magidson, J. y Vermunt, J. K. (2004). Latent class models. En D. Kaplan (Ed.), The Sage Handbook of Quantitative Methodology for the Social Sciences (pp. 175-198). Thousand Oakes: Sage Publications.

Ministerio de Educación de Chile. (2009). Estándares TIC para la Formación Inicial Docente en el Contexto Chileno: Estrategias para su difusión y adopción. Santiago: Ministerio de Educación de Chile.

Ministerio de Educación de Chile. (2003). Marco para la Buena Enseñanza. Recuperado de http://www.rmm.cl/usuarios/equiposite/ doc/200312031457060.mbe.pdf , visitado en agosto de 2012.

Nussbaum, M., Weitz, J., Sibils, X., Díaz, A. y Claro, M. (2012) Evaluación de la Implementación de la Estrategia Laboratorios Móviles Computacionales (LMC). Informe Final Proyecto FONIDE No511051.

Salinas, A., Sánchez, J., Purcell, O. y Mendoza, C. (2009). Factores que inciden en el desarrollo y sustentabilidad de prácticas innovadoras de integración curricular de tecnologías de la información (TICs) en la sala de clases en profesores de Enseñanza Media. Informe Final Proyecto FONIDE F310837-2008.

Salinas, A., Ruiz, P., Vergara, A., González, C., Raquimán, P. y Gértudrix, F. (2012). Participación en redes profesionales digitales e innovación en las prácticas docentes en la sala de clases. Informe Final Proyecto FONIDE F511068-2010.

Scheihing, E., Casanova, R., Contreras, P. y Arancibia, M. (2011). Diseños Didácticos Colaborativos Para Aprender En La Escuela Utilizando Recursos de la web 2.0. I Congreso Internacional de Metodologías de Aprendizaje Colaborativo a través de las TIC, Actas CIMAC 2011, Salamanca, Junio 2011.

Scheihing, E., Aros, C. y Guerra, J. (2012). Analyzing the Behavior of a Teacher Network in a Web 2.0 environment. Actas EDM2012, Grecia, Junio 2012.

Sigalès, C., Mominó, J., Meneses, J. y Badia, A. (2009). La integración de internet en la educación escolar española. Barcelona: Ariel. 